\title{
A panoramic view of the Southern quadrant of the Andromeda galaxy outer halo
}

\author{
Nicolas F. Martin ${ }^{1}$, Rodrigo A. Ibata ${ }^{2}$ and Mike J. Irwin ${ }^{3}$ \\ ${ }^{1}$ Max-Planck Institute für Astronomie, Königstuhl 17, D-69117 Heidelberg, Germany \\ email: martin@mpia-hd.mpg.de \\ ${ }^{2}$ Observatoire de Strasbourg, 11 rue de l’Université, F-67000 Strasbourg, France \\ email: ibata@astro.u-strasbg.fr \\ ${ }^{3}$ Institute of Astronomy, Madingley Road, Cambridge CB3 0HA, U.-K. \\ email: mike@ast.cam.ac.uk
}

\begin{abstract}
How clumpy are galactic halos? Recent observations around both the Milky Way and the Andromeda galaxy (M31) have revealed numerous faint stellar streams and dwarf galaxies, leading to the belief that more of these may yet remain undetected. In this contribution, we present the map produced from the Megacam/CFHT survey that our group has undertaken in the outer halo of M31 and that, for the first time, gives a deep panoramic view of a significant region of the outer halo of a spiral galaxy. This panoramic survey, which covers $\sim 60$ sq. deg. of the southern quadrant of the M31 halo, extends the WFC/INT survey of the inner halo (Ferguson et al. 2002) from a projected distance of $\sim 50$ to $\sim 150 \mathrm{kpc}$. It is deep enough to cover three magnitudes below the tip of the red giant branch of stellar populations at the distance of M31. The survey reveals:

- Three faint dwarf galaxies with absolute magnitudes in the range $-7.3<M_{V}<-6.4$ and the most remote M31 globular cluster at a projected distance of $\sim 120 \mathrm{kpc}$ from M31 (see Martin et al. 2006 for more details).

- That the giant stream of Ibata et al. (2001) covers a much wider area than previously expected from shallower surveys, has an apocenter at $125 \pm 25 \mathrm{kpc}$ from M31 and is probably due to the accretion of a small disk galaxy.

- A new stellar stream or shell approximately perpendicular to the minor axis of M31 at a projected distance of $120 \mathrm{kpc}$ and with a metallicity of $[\mathrm{Fe} / \mathrm{H}] \sim-1.5$ (assuming it is at the distance of M31).

- A new stellar stream along the ma jor axis of M31 that extends to at least $100 \mathrm{kpc}$ from M31 with $[\mathrm{Fe} / \mathrm{H}] \sim-1.3$ (once again assuming it is at the distance of M31).

- Regions void of any stellar structure brighter than $34-35 \mathrm{mag} / \operatorname{arcsec}^{2}$ at a distance of 100 to $130 \mathrm{kpc}$ from M31.

The survey shows that the outer halo of the Andromeda galaxy is very structured, in qualitative agreement with recent cosmological simulations (e.g. Bullock \& Johnston, 2005).
\end{abstract}

Keywords. galaxies: evolution, galaxies: halos, galaxies: individual (M31), galaxies: structure, galaxies: Local Group

We have performed a matched filter analysis (e.g. Rockosi et al. 2002) of the survey to minimize the influence of foreground and background contaminants (mainly Milky Way dwarf stars and compact galaxies respectively) and enhance the features that are present in the halo of M31. The map that is produced in this way is presented on the top panel of Figure 1. In the bottom panel, a sketch of the survey helps identify the features listed in the abstract.

The survey is presented in more details in Ibata et al. (2007). 

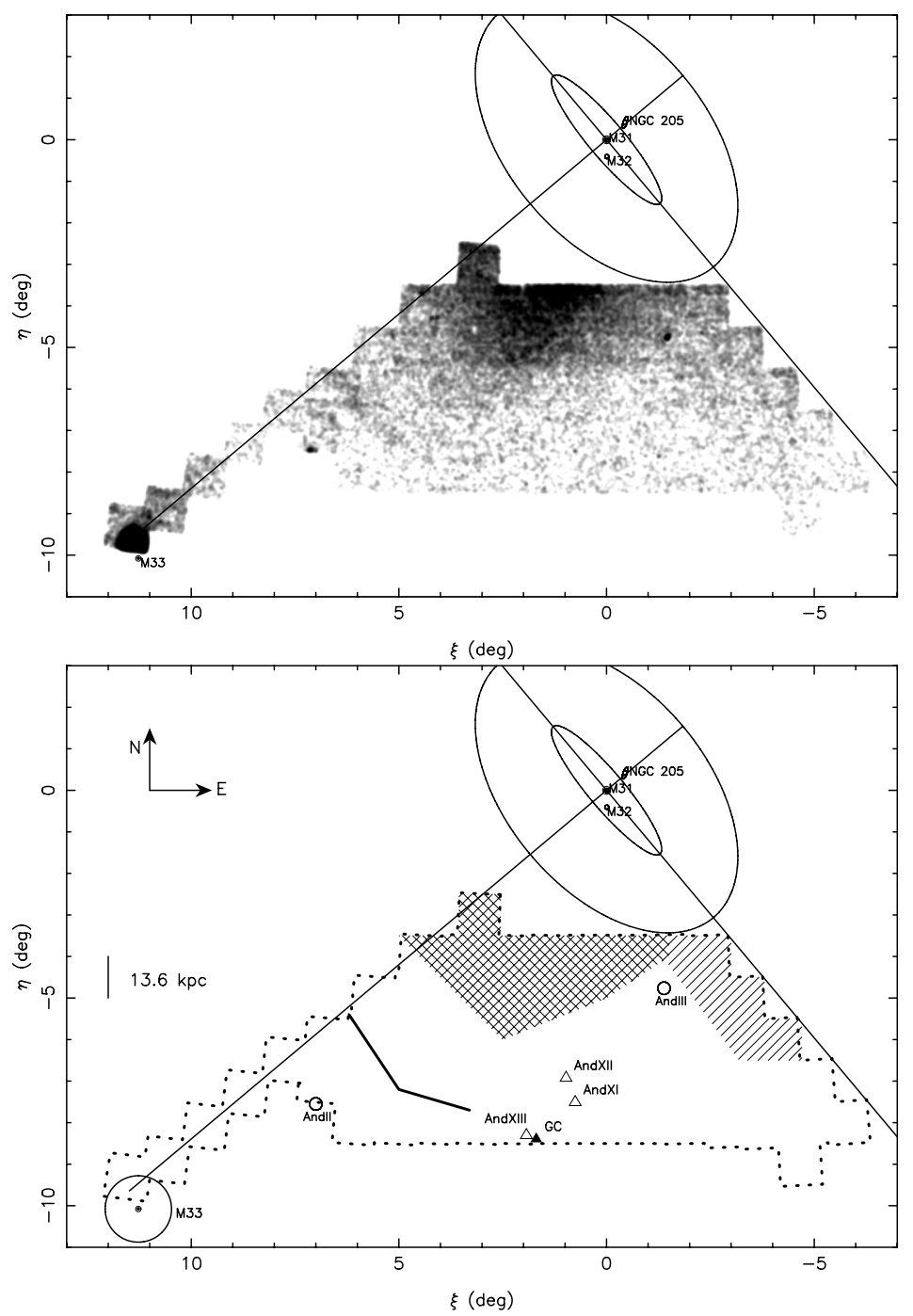

Figure 1. Top: matched-filter map of the MegaCam/CFHT survey. North is to the top and East to the right. Bottom: Cartoon highlighting the features present in the survey (delimited by the dotted line). Previously known M31 satellites are shown as circles (And II, And III and M33), the three faint dwarf galaxies And XI, And XII and And XIII as empty triangles and the most remote M31 globular cluster as a filled triangle (see Martin et al. 2006). The extent of the giant stream discovered by Ibata et al. (2001) is represented by the cross-hatched region, that of the new ma jor axis stream by the hatched region and the stream near the minor axis of M31 by the thick line. The physical scale at the distance of M31 is given on the left.

\section{References}

Bullock J. S. \& Johnston K. V. 2005, ApJ 635, 931

Ferguson A. M. N., Irwin M. J., Ibata R. A., Lewis G. F. \& Tanvir N. R. 2002, AJ 124, 1452

Ibata R., Irwin M., Lewis G., Ferguson A. M. N. \& Tanvir N. 2001, Nature 412, 49

Ibata R. A., Martin N. F., Irwin M. J., Chapman S. C., Lewis G. F., Ferguson A. M. N. \& Tanvir N. R. 2007, in preparation

Martin N. F., Ibata R. A., Irwin M. J., Chapman S. C., Lewis G. F., Ferguson A. M. N. \& Tanvir N. R. 2006, MNRAS 371, 1983

Rockosi et al. 2002, AJ 124, 349 